Kansas State University Libraries

New Prairie Press

\title{
STATISTICAL ANALYSIS SOFTWARE FOR MULTIPLICATIVE INTERACTION MODELS
}

Eun-Joo Lee

Dallas E. Johnson

Follow this and additional works at: https://newprairiepress.org/agstatconference

Part of the Agriculture Commons, and the Applied Statistics Commons

\section{(c) (1) $\Theta(9$}

This work is licensed under a Creative Commons Attribution-Noncommercial-No Derivative Works 4.0 License.

\section{Recommended Citation}

Lee, Eun-Joo and Johnson, Dallas E. (2004). "STATISTICAL ANALYSIS SOFTWARE FOR MULTIPLICATIVE INTERACTION MODELS," Conference on Applied Statistics in Agriculture. https://doi.org/10.4148/

2475-7772.1156

This is brought to you for free and open access by the Conferences at New Prairie Press. It has been accepted for inclusion in Conference on Applied Statistics in Agriculture by an authorized administrator of New Prairie Press. For more information, please contact cads@k-state.edu. 


\title{
STATISTICAL ANALYSIS SOFTWARE FOR MULTIPLICATIVE INTERACTION MODELS
}

\author{
Eun-Joo Lee \\ Advanced Micro Devices Inc., Sunnyvale, CA 94088-3453 \\ and \\ Dallas E. Johnson \\ Kansas State University, Manhattan, KS 66506-0802
}

\begin{abstract}
In a two-way cross-classified experiment, one is almost always interested in whether the two factors interact or not. When there are no independent replications, there are no traditional tests for interaction. This research considers the problem of analyzing a two-way cross-classified experiment using multiplicative interaction models when there are no independent replications and interaction between the two factors may exist. The purpose of this research is to develop SAS ${ }^{\circledR}$ macros to provide user-friendly statistical software for the analysis of interaction in two-way experiments. The macros also provide many useful graphical displays including displays to help one determine the pattern of interaction when a pattern exists and to help one interpret the results of the analyses.
\end{abstract}

KEYWORDS: Two-Way experiments, AMMI, Genotype-by-Environmental interaction.

\section{INTRODUCTION}

In a two-way cross-classified experiment, one is almost always interested in whether the two factors interact or not. When there are no independent replications, there are no traditional tests for interaction. This research considers the problem of analyzing a two-way cross-classified experiment when there are no independent replications and interaction between the two factors may exist.

There are some experiments that are impossible to replicate because of their own attributes. In examining human genotype-by-environmental interaction in an analysis of individual differences in human populations, one can only assess the performance of any given array of human genotypes within a single culture, at a single point in time. Also, genotype-by-environmental interactions can occur when various plant genotypes are grown across diverse environments as in a plant breeding study, but it is not possible to replicate environments, such as specific years and/or specific locations, in this kind of an experiment. The additive nature of the ordinary ANOVA technique allows us to describe main effects, but the interaction between genotypes and environments is completely confounded with the experimental error since the residuals from an additive model contain information about both interaction and the experimental error. If interaction exists only in a part of a two-way cross-classified layout and one can determine the pattern of interaction, then it would be possible to partition the residual sum of squares from the additive model into a portion that is free from interaction (i.e. estimates the experimental error variance) and a portion that estimates interaction effects.

Rating scales are one of the most popular judgmental measures used by one group of individuals to judge one or more aspects of other individuals, such as job performance appraisal, personnel selection based on interviews, teachers' ratings of their students' behavior, and judges' ratings of participants in events like figure skating, diving, and gymnastics in Olympic games. Rating scales are widely used in business, behavioral science, and sports as a useful assessment technique. One can view the data from the above situations as two-way cross-classified experiments without replication.

Many experiments are very expensive to conduct so that researchers are often forced to limit the number of treatment combinations that can be studied in order to be able to replicate the treatment combinations so as to get an independent estimate of the experimental error variance. If the major 
objective of the experiment is exploratory rather than confirmatory, it may be more desirable to study many different treatment combinations, each performed once, rather than a few treatment combinations each replicated many times.

Clearly, there exist needs to analyze data from these types of experiments and methods based on multiplicative interaction models to solve these kinds of problems have been proposed during the last few decades. However, using these methods requires complex matrix calculation and no generalized software is readily available to experimenters to make use of these methods easily. Currently, the models are usable only by a small number of researchers who can write their own programs using SAS/IML ${ }^{\circledR}$, S-PLUS ${ }^{\circledR}$, or MATLAB $^{\circledR}$ for each analysis. The purpose of the research described in this paper is to develop SAS ${ }^{\circledR}$ macros that will provide user-friendly statistical software for the analysis of interaction in two-way nonreplicated experiments. In addition to performing the complex calculations required to fit multiplicative interaction models, the software provides many useful graphical displays that will enable large numbers of researchers to easily interpret the results of their statistical analyses. The macros also output the analyses in a user-friendly format.

\section{ANALYSES OF TWO-WAY NONREPLICATED EXPERIMENTS}

The AMMI (Additive Main-effects and Multiplicative Interaction) models allow one to analyze twoway data with interaction along with a traditional additive main effects framework even if there are no independent replications. Multiplicative interaction models are non-traditional nonlinear models that allow one to analyze many nonreplicated experiments. Gollob (1968) and Mandel (1969) independently considered a multiplicative interaction model of the form

$$
y_{i j}=\mu+\tau_{i}+\beta_{j}+\lambda_{1} \alpha_{1 i} \gamma_{1 j}+\lambda_{2} \alpha_{2 i} \gamma_{2 j}+\cdots+\lambda_{k} \alpha_{k i} \gamma_{k j}+\varepsilon_{i j}, \quad i=1,2, \cdots, t ; j=1,2, \cdots, b
$$

for some $k \leq b-1$ where it is assumed that $\varepsilon_{i j} \sim$ i.i.d. $N\left(0, \sigma^{2}\right)$ and, without loss of generality, that $b \leq t$. The following restrictions are imposed on the parameters without any loss of generality:

$$
\begin{aligned}
& \sum_{i} \tau_{i}=\sum_{j} \beta_{j}=0, \quad\left|\lambda_{1}\right| \geq\left|\lambda_{2}\right| \geq \cdots \geq\left|\lambda_{k}\right|, \\
& \sum_{i} \alpha_{r i}=\sum_{j} \gamma_{r j}=0 \text { for } r=1,2, \cdots, k, \\
& \sum_{i} \alpha_{r i}^{2}=\sum_{j} \gamma_{r j}^{2}=1 \text { for } r=1,2, \cdots, k \text {, and } \\
& \sum_{i} \alpha_{r i} \alpha_{r^{\prime} i}=\sum_{j} \gamma_{r j} \gamma_{r^{\prime} j}=0 \text { for } r \neq r^{\prime}=1,2, \cdots, k .
\end{aligned}
$$

The above assumptions are made only to provide parameter identifiability in model (2.1). Gollob assumed a replicated experiment and, thus had an independent estimate of the error variance, whereas Mandel assumed one observation per treatment combination. The main difference between Gollob's and Mandel's approach is the number of degrees of freedom that are assigned to each of the multiplicative interaction terms.

Tukey (1949) was the first to propose a test for interaction in the two-way treatment structure experiment with one observation per treatment combination. Tukey did not specify any particular form of interaction in the model when he proposed the test. Hegemann and Johnson (1976) showed that his test has relatively good power when the interaction term is a scalar multiple of the product of the two main effects, i.e., when 


$$
y_{i j}=\mu+\tau_{i}+\beta_{j}+\lambda \tau_{i} \beta_{j}+\varepsilon_{i j}, \quad i=1,2, \cdots, t ; \quad j=1,2, \cdots, b .
$$

Model (2.2) is called Tukey's model and it is a special case of (2.1) with $k=1, \alpha_{i}=\tau_{i} \forall i$, and $\gamma_{j}=\beta_{j} \forall j$. A test for interaction is made by testing $H_{0}: \lambda=0$ vs $H_{A}: \lambda \neq 0$ in model (2.2). See Graybill (2000) for proofs. If one fails to reject $H_{0}$, then there is no interaction of the form $\lambda \tau_{i} \beta_{j}$, but this does not rule out existence of other forms of interaction. In addition, Hegemann and Johnson (1976) showed that Tukey's test is not an unbiased test, i.e., the probability of rejecting $H_{0}: \lambda=0$ when interaction exists could be less than the probability of rejecting when there is no interaction.

Mandel (1961) generalized Tukey's model to

$$
y_{i j}=\mu+\tau_{i}+\beta_{j}+\alpha_{i} \beta_{j}+\varepsilon_{i j}, \quad i=1,2, \cdots, t ; \quad j=1,2, \cdots, b .
$$

Model (2.3) is a special case of (2.1) when $k=1$ and $\lambda \gamma_{j}=\beta_{j} \forall j$ and Tukey's model (2.2) is a special case of (2.3) when $\alpha_{i}=\lambda \tau_{i} \forall i$. Mandel's model is often called a 'bundle-of-straight-lines' model because an alternative form of model (2.3) is $y_{i j}=\mu+\tau_{i}+\left(1+\alpha_{i}\right) \beta_{j}+\varepsilon_{i j}$ and $y_{i j}$ is a linear function of $\beta_{j}$ for each value of $i$ with a different slope parameter $\left(1+\alpha_{i}\right)$ for each line. Both Tukey's and Mandel's models are special cases of the multiplicative interaction model. Mandel's model reduces to an additive model if $\alpha_{1}=\alpha_{2}=\cdots=\alpha_{t}$, therefore a test for interaction can be obtained by testing $H_{0}: \alpha_{1}=\alpha_{2}=\cdots=\alpha_{t}$. See Mandel (1961) for proofs. Again, if $H_{0}$ is not rejected, one can only say that there is likely no interaction of the form given by Mandel's model. Another form of Mandel's model is $y_{i j}=\mu+\tau_{i}+\beta_{j}+\tau_{i} \gamma_{j}+\varepsilon_{i j}, \quad i=1,2, \cdots, t ; j=1,2, \cdots, b$. In this model a test for interaction is made by testing $H_{0}: \gamma_{1}=\gamma_{2}=\cdots=\gamma_{b}$.

When an experimenter wishes to use a multiplicative interaction model, it is necessary to determine how many multiplicative interaction terms are required to adequately model the data. It is desirable to choose a model having as few terms as possible while adequately modeling the two-way data. Yochmowitz and Cornell (1978) gave a stepwise procedure for determining the number of terms necessary to explain the interaction. However, Milliken and Johnson (1989) noted that "... in real data situations, an experiment requiring more than two terms has never been encountered, and in most cases only one term has been required."

\subsection{Type I and Type II Interaction Plots}

Two different types of interaction plots called a Type I interaction plot and a Type II interaction plot for a set of two-way cell mean parameters are illustrated by Milliken and Johnson (1989). A Type I interaction plot is constructed by plotting $\mu_{i j}$ against $i$ (or $j$ ), for each possibility of the other treatment factor, $B_{j}$ (or $T_{i}$ ). A Type II interaction plot is constructed by plotting $\mu_{i j}$ against the maineffect parameters of one treatment factor, $\tau_{i}$ (or $\beta_{j}$ ), for each possibility of the other treatment factor, $B_{j}$ (or $T_{i}$ ). Four different models, an additive two-way model, Tukey's model, Mandel's bundle-ofstraight-lines model, and the AMMI model, are considered by the SAS ${ }^{\circledR}$ macros developed in this research and each is examined through Type I and Type II interaction plots in this section. The Type II plots given in this Section take $\tau_{i}=\bar{\mu}_{i .}-\bar{\mu}_{. .}, i=1,2,3,4$.

When the cell mean parameters are from an additive two-way model, a Type I interaction plot always consists of line segments which are parallel to one another and a Type II interaction plot always consists of parallel lines rather than parallel line segments. Figure 1 shows a Type I and Type II interaction plot for cell mean parameters from an additive model.

Figure 2 shows a set of cell mean parameters satisfying Tukey's model (2.2) and their Type I and Type 
II interaction plots. An examination of Type I interaction plot reveals little more other than the two factors interact. However, the Type II interaction plot from Tukey's model reveals a plot consisting of several straight lines all intersecting in a single point. This always holds for data that can be modeled exactly by Tukey's model.

Figure 3 shows a set of cell mean parameters satisfying Mandel's model (2.3) and Type I and Type II interaction plots for these cell means. Again, an examination of the Type I interaction plot does not provide much information other than these cell mean parameters are from a nonadditive model. The Type II interaction plot is much more informative when the cell means are from Mandel's model. An examination of the Type II interaction plot in Figure 3 suggests that $\mu_{i j}$ is a linear function of $\hat{\tau}_{i}$ for each level of $B_{j}$ with a different slope parameter for each line. This is why Mandel's model is called a 'bundle-of-straight-lines' model.

For data that can be modeled by Tukey's model, it does not matter whether the $\mu_{i j}$ 's are plotted against $\tau_{i}$ or plotted against $\beta_{j}$. In either case, the resulting Type II interaction plot consists of straight lines that intersect in a single point. However, for data that can be modeled by Mandel's model, the fact that $\mu_{i j}$ can be expressed as a linear function of $\hat{\tau}_{i}$ for each level of $B_{j}$ as shown in Figure 3 does not guarantee that $\mu_{i j}$ can also be expressed as a linear function of $\hat{\beta}_{j}$ for each level of $T_{i}$. An appropriate form of the model should be carefully chosen when selecting one of Mandel's models.

The cell means given in Figure 4 are obtained from an AMMI model (2.1) with one interaction term by letting $\mu=29, \quad \boldsymbol{\tau}^{\prime}=\left[\begin{array}{llll}-10 & 4 & -2 & 8\end{array}\right], \quad \boldsymbol{\beta}^{\prime}=\left[\begin{array}{lllll}-5 & 2 & 4 & 0 & -1\end{array}\right], \quad \lambda=354.9648, \quad \boldsymbol{\alpha}^{\prime}=\left[\begin{array}{lllll}0 & -.267 & -.535 & .802 & 0\end{array}\right]$, and $\gamma^{\prime}=\left[\begin{array}{lll}.632 & -.316 & \text {.316 -.632] }\end{array}\right.$. The Type I and Type II interaction plots for these cell means are also given in Figure 4. Neither the Type I nor Type II interaction plot reveals any interesting characteristics of the cell means in this case.

\subsection{Maximum Likelihood Estimators}

Johnson and Graybill (1972) obtained the maximum likelihood estimators of the parameters in model (2.1) when $k=1$. The maximum likelihood estimators of the parameters in model (2.1) are

$$
\begin{aligned}
& \hat{\mu}=\bar{y}_{. .} ; \\
& \hat{\tau}_{i}=\bar{y}_{i .}-\bar{y}_{. .}, \quad i=1,2, \cdots, t ; \\
& \hat{\beta}_{j}=\bar{y}_{. j}-\bar{y}_{. .}, \quad j=1,2, \cdots, b ; \\
& \hat{\lambda}_{r}^{2}=l_{r}, \quad r=1,2, \cdots, k ; \\
& \tilde{\sigma}^{2}=\frac{\sum_{i j} z_{i j}^{2}-\sum_{r=1}^{k} l_{r}}{t b}
\end{aligned}
$$

where $l_{1}>l_{2}>\cdots>l_{k}>l_{k+1}>\cdots>l_{b-1}$ are the non-zero characteristic roots of $\mathbf{Z}^{T} \mathbf{Z}$ or $\mathbf{Z Z}^{T}$, where $\mathbf{Z}=\left(z_{i j}\right)$ and $z_{i j}=y_{i j}-\bar{y}_{i .}-\bar{y}_{. j}+\bar{y}_{. .}, \hat{\boldsymbol{\alpha}}_{r}$ is the normalized characteristic vector of $\mathbf{Z Z}^{T}$ corresponding to the characteristic root, $l_{r}, \quad r=1,2, \cdots, k$, and $\hat{\gamma}_{r}$ is the normalized characteristic vector of $\mathbf{Z}^{T} \mathbf{Z}$ corresponding to the characteristic root, $l_{r}, r=1,2, \cdots, k$. The proper sign for $\hat{\lambda}_{r}$ is obtained by taking $\hat{\lambda}_{r}=\hat{\boldsymbol{\alpha}}_{r}^{T} \mathbf{Z} \hat{\boldsymbol{\gamma}}_{r}, \quad r=1,2, \cdots, k$. The maximum likelihood estimator $\tilde{\sigma}^{2}$ is not an unbiased estimate of $\sigma^{2}$. An estimator of $\sigma^{2}$ that is less biased is 


$$
\tilde{\sigma}^{2}=\frac{\sum_{i j} z_{i j}^{2}-\sum_{r=1}^{k} l_{r}}{(t-1)(b-1)-\sum_{r=1}^{k} v_{r}}
$$

where $v_{r}=E_{\lambda_{1}=\lambda_{2}=\cdots=\lambda_{r}=0}\left[l_{r} / \sigma^{2}\right]$.

Milliken and Johnson (1989) showed that an examination of the interaction parameter estimates reveals information about the nature of the interaction in a set of two-way means. Suppose that the values of some of the $\hat{\alpha}_{r i}$ 's in model (2.1) are nearly equal for $r=1,2, \cdots, k$. This suggests that the corresponding rows do not interact with the column treatments. Similarly, when values of some of the $\hat{\gamma}_{r j}$ 's are nearly equal for $r=1,2, \cdots, k$, then the corresponding columns do not likely interact with the row treatments. This situation is easy to see when $k=1$, and may be easy to see when $k=2$. When $k>2$, it is still worth considering, although it may be hard to determine the nature of the interaction in such cases.

\subsection{Testing Interaction Hypotheses}

Mandel (1969) proposed an approximate size $\alpha$ test for interaction in the AMMI model when $k=1$ as reject $H_{0}: \lambda_{1}=0$ in favor of $H_{A}: \lambda_{1} \neq 0$ if

$$
F^{*}=\frac{l_{1} / v_{1}}{\hat{\sigma}^{2}}>F_{\alpha, v_{1},(t-1)(b-1)-v_{1}}
$$

where $v_{1}=E_{\lambda_{1}=0}\left[l_{1} / \sigma^{2}\right]$. That is, Mandel suggested that $l_{1} / \sigma^{2}$ is approximately $\chi^{2}\left(v_{1}\right)$ if $H_{0}: \lambda_{1}=0$ is true, $(t-1)(b-1) \hat{\sigma}^{2} / \sigma^{2}$ is approximately $\chi^{2}\left((t-1)(b-1)-v_{1}\right)$, and that $l_{1}$ and $\hat{\sigma}^{2}$ are approximately independent. Thus, $F^{*}=l_{1} /\left(v_{1} \hat{\sigma}^{2}\right)$ is approximately $F\left(v_{1},(t-1)(b-1)-v_{1}\right)$ if $H_{0}: \lambda_{1}=0$ is true. Mandel's test statistic was shown to be a likelihood ratio test statistic for testing $H_{0}: \lambda_{1}=0$ by Johnson and Graybill (1972). The SAS $^{\circledR}$ macros developed in this paper simulate values for $v_{1}$ for various choices of $t$ and $b$.

In the case where $k>1$, Mandel (1969) proposed an ANOVA table of the form in Table 1. To test $H_{0}: \lambda_{r}=0, \lambda_{1} \neq 0, \lambda_{2} \neq 0, \cdots, \quad \lambda_{r-1} \neq 0$ vs $H_{A}: \lambda_{r} \neq 0, \lambda_{1} \neq 0, \lambda_{2} \neq 0, \cdots, \lambda_{r-1} \neq 0$, one uses

$$
F_{r}^{*}=\frac{l_{r} / v_{r}}{\left(\sum_{i j} z_{i j}^{2}-\sum_{i=1}^{r-1} l_{i}\right) /\left((t-1)(b-1)-\sum_{i=1}^{r-1} v_{i}\right)}
$$

and one rejects $H_{0}$ at the $\alpha \times 100 \%$ significance level if $F_{r}^{*}>F_{\alpha, v_{r},(t-1)(b-1)-\sum_{i=1}^{r-1} v_{i}}$. The $\mathrm{SAS}^{\circledR}$ macros produced simulated values of $v_{1}, v_{2}, \cdots, v_{k}$ and an ANOVA table similar to Table 1 except that all F-values and p-values are based on $\hat{\sigma}^{2}$ in (2.5) with degrees of freedom $(t-1)(b-1)-\sum_{i=1}^{r-1} v_{i}$.

If two treatments interact, experimenters also want to know where the interaction exists in the data. Marasinghe and Johnson (1982) derived a likelihood ratio statistic for testing $H_{0}: \mathbf{H} \boldsymbol{\alpha}=\mathbf{0}$ and $\mathbf{G} \boldsymbol{\gamma}=\mathbf{0}$ vs $H_{0}: \mathbf{H} \boldsymbol{\alpha} \neq \mathbf{0}$ or $\mathbf{G} \boldsymbol{\gamma} \neq \mathbf{0}$, where $\mathbf{H}$ is any $p \times t$ matrix of rank $p$ whose rows are contrasts and $\mathbf{G}$ is any $q \times b$ matrix of rank $q$ whose rows are contrasts when $k=1$ in model (2.1). The test statistic is given by 


$$
\Lambda=\frac{\sum_{i j} z_{i j}^{2}-l_{1}}{\sum_{i j} z_{i j}^{2}-l_{1}^{* *}}
$$

where $l_{1}^{* *}$ is the largest characteristic root of

$$
\left[\mathbf{I}-\mathbf{H}^{T}\left(\mathbf{H} \mathbf{H}^{T}\right)^{-1} \mathbf{H}\right] \mathbf{Z}\left[\mathbf{I}-\mathbf{G}^{T}\left(\mathbf{G G}^{T}\right)^{-1} \mathbf{G}\right] \mathbf{Z}^{T}\left[\mathbf{I}-\mathbf{H}^{T}\left(\mathbf{H H}^{T}\right)^{-1} \mathbf{H}\right] .
$$

The hypothesis $H_{0}: \mathbf{H \alpha}=\mathbf{0}$ and $\mathbf{G} \boldsymbol{\gamma}=\mathbf{0}$ is rejected for small values of $\Lambda$ and the null distribution of $\Lambda$ depends on $b, t, p, q$, and $\delta=\lambda_{1} / \sigma$. This test can be used to test for the equality of subsets of the $\alpha$ 's and the $\gamma$ 's individually or simultaneously which implies that the corresponding sub-tables in the data are additive. Hence, the test can be used to identify such sub-tables. If there is no significant interaction within selected sub-tables of the data, then the experimenter may use this information to obtain a more reliable estimate of the experimental error variance.

Johnson and Graybill (1972) showed that the non-zero characteristic roots of $\mathbf{Z}^{T} \mathbf{Z}$ are distributed as the characteristic roots of a noncentral Wishart matrix $\mathbf{W} \sim W_{b-1}\left(t-1, \sigma^{2} \mathbf{I}, \mathbf{M}\right)$ where $\mathbf{M}=\mathbf{K}^{T} \boldsymbol{\Gamma}^{T} \boldsymbol{\Gamma} \mathbf{K}$, $\boldsymbol{\Gamma}=\left[\lambda_{1} \alpha_{1 i} \gamma_{1 j}\right]$, and $\mathbf{K}$ is any $b \times(b-1)$ matrix satisfying $\mathbf{K K}^{T}=\mathbf{I}_{b}-(1 / b) \mathbf{J}_{b}$ and $\mathbf{K}^{T} \mathbf{K}=\mathbf{I}_{b-1}$. For the case when $b=3$, Johnson (1974) found the mean and variance of the characteristic roots of a noncentral Wishart matrix. Other than this special case, the moments of the characteristic roots of a Wishart matrix are not explicitly known.

\section{AMMI MACROS FOR ANALYZING MULTIPLICATIVE INTERACTION MODELS}

This section introduces $\mathrm{SAS}^{\circledR}$ macros to help analyze two-way cross-classified experiments with no independent replications developed under the SAS ${ }^{\circledR}$ system release 8.2 (TS2MO) on a windows environment. This developed set of macros is called the AMMI macros. The AMMI macros consist of six independently executable macros and 22 sub-macros that are called by the six main macros. The AMMI macros provide three stages of analytic tools; pre-analysis for diagnosing interaction, model fitting for selecting a suitable model, and testing interaction contrasts for finding patterns of interaction in the data.

All plots produced by the AMMI macros are generated in an Adobe ${ }^{\circledR}$ Portable Document Format (PDF) file before they are shown in the SAS ${ }^{\circledR}$ graph window. Each page of a PDF file may be exported to another type of image file using Adobe ${ }^{\circledR}$ Acrobat $^{\circledR}$. Exporting graphic output as image files with Adobe ${ }^{\circledR}$ Acrobat ${ }^{\circledR}$ gives high quality graphs needed for publication purposes. Most of the SAS ${ }^{\circledR}$ graphic output loses its original quality when exported to other types of image files. The PDF graphic output has the unique property that one gets the same quality of graphic output as seen in the SAS ${ }^{\circledR}$ graph window. The following sections explain the methods employed to build the AMMI macros by their functions.

\subsection{Pre-analysis}

The macro \%PreviewAMMI is designed to explore and diagnose interactions between the two factors. After a data set is ready for analysis, the user has options to display the data graphically for diagnostic purposes. Type I interaction plots and Type II interaction plots for a set of two-way cell responses as described in Milliken and Johnson (1989) are available. Another way to think about the AMMI model is that it provides a singular value decomposition of the matrix of residuals, or equivalently, it provides a principal component analysis of the matrix $\mathbf{Z}^{T} \mathbf{Z}$ and/or $\mathbf{Z Z}^{T}$. Thus, one method that can be used to guide one in the choice of the number of interaction terms is a scree plot of the characteristic roots of 
$\mathbf{Z}^{T} \mathbf{Z}$ and/or $\mathbf{Z Z}^{T}$. A scree plot plots the values of the characteristic roots against the order of the characteristic roots. That is, one plots the pairs $\left(1, l_{1}\right),\left(2, l_{2}\right), \cdots,\left(b-1, l_{b-1}\right)$. When the points on the graph tend to level off, these characteristic roots are usually close enough to zero that the corresponding multiplicative interaction terms can be ignored. The \%PreviewAMMI macro provides a scree plot of the non-zero characteristic roots of the matrix $\mathbf{Z}^{T} \mathbf{Z}$ and/or $\mathbf{Z Z}^{T}$ where $\mathbf{Z}$ is the residual matrix obtained after fitting an additive model to the two-way data. This helps to determine the number of multiplicative interaction terms required to adequately model the data. In addition to diagnostic graphics, a table of all $2 \times 2$ interaction contrasts calculated from all possible pairwise row and pairwise column contrasts is provided to help one identify the pattern of interaction, should a pattern exist.

\subsection{Fitting Models}

The macro \%FitAMMImodel is designed to fit two-way data in one or more of several different models. Four different models can be fitted, individually or simultaneously; these are an additive model, Tukey's model, Mandel's bundle-of-straight- lines models, and an AMMI model. When Tukey's or Mandel's model is chosen, a fitted line plot with data points will be provided along with ANOVA tables. As shown in Section 2, Tukey's model can be viewed as a special case of Mandel's model when the lines intersect in a common point. The sum of squares for Mandel's interaction term can be divided into two parts; the sum of squares for Tukey's interaction term and the remainder. When the remainder is small then Mandel's model provides no better fit to the data than that given by Tukey's model. In this case, one says that lines are 'concurrent'. That is, the lines intersect in a single point. When a Mandel's model is chosen for fitting, a test for concurrency is provided prior to fitting Mandel's model. The test for concurrency is a test that the lines intersect in a common point. Additional functions of the AMMI macros related to Tukey's and Mandel's models are discussed later. After fitting each model, an output dataset is created with predicted values and residuals appended to the original input data.

Pseudo F-tests are provided for each multiplicative interaction term in the AMMI models as illustrated by Table 1. When an AMMI model is fit, the expected values of the characteristic roots of $\mathbf{Z}^{T} \mathbf{Z}$ are required for different values of $t$ and $b$ in order to conduct statistical tests on the multiplicative interaction terms. The exact moments of the characteristic roots are known for the case when $b=3$. See Johnson (1974) for details. When $b>3$, the expected values of the characteristic roots are simulated by the macro \%FitAMMImodel to assign degrees of freedom to each of the multiplicative interaction terms. Estimates of the moments of the characteristic roots of $\mathbf{Z}^{T} \mathbf{Z}$ are obtained by generating 999 random matrices having a central Wishart distribution with covariance matrix equal to the identity matrix and calculating the characteristic roots of each. The expected value of the sum of the characteristic roots is $(t-1)(b-1)$ since $E\left(l_{1}+l_{2}+\cdots+l_{b-1}\right)=E(R S S)=(t-1)(b-1)$. The sum of the empirical moments of the characteristic roots of $\mathbf{Z}^{T} \mathbf{Z}$ is not equal to $(t-1)(b-1)$. When the expected values are overestimated, the sum of simulated moments is generally greater than $(t-1)(b-1)$. Similarly, when the expected values are underestimated, the sum of simulated moments is generally less than $(t-1)(b-1)$. Consequently, a bias correction is made by multiplying each simulated moment by $(t-1)(b-1)$ and then dividing by the mean of the 999 residual sums of squares of the simulated moments from an additive model. Whether the original estimates are biased upwards or downwards, estimates become less biased after the bias correction.

A previously simulated set of estimates for $E_{\lambda_{1}=\lambda_{2}=\cdots=\lambda_{b-1}=0}\left[l_{i}\right], \quad i=1,2, \cdots, b-1$ is provided as a SAS $^{\circledR}$ dataset named AMMI.EX_L with the AMMI macros. The dataset AMMI.EX_L is referenced, updated, and expanded by the macro \%FitAMMImodel to assign degrees of freedom to each of the multiplicative interaction terms in AMMI models. When an AMMI model is chosen, one can specify the number of multiplicative interaction terms required to adequately model the data. There are four options to choose 
the method to calculate a set of degrees of freedom associated with multiplicative interaction terms: (1) find a set of values from a previously simulated table, (2) run a simulation to get a set of degrees of freedom for the case under considerations, (3) run a simulation and store the simulated values in the table described in (1), or (4) use Gollob's method, which assigns $(b+t-1-2 m)$ degrees of freedom to the $m$ th multiplicative interaction term. This paper recommends that analysts use simulated moments as Gollob's method gives highly biased estimates of the degrees of freedom associated with each interaction term.

\subsection{Testing Interaction Contrasts}

Once it has been determined, that there is interaction in the data, one may want to find combinations of the two factors that are responsible for the interaction and to know whether a few selected treatment combinations are responsible for all or most of the interaction in the two-way data.

The macro \%IC2by2T is designed to test all $2 \times 2$ interaction contrasts calculated from all possible pairwise row and pairwise column contrasts and based on a given error variance along with its corresponding degrees of freedom. This is a model-free method to check if certain combinations of treatment effects are not responsible for the interaction. This module is built to provide tests based on information from the selected model in addition to calculating all $2 \times 2$ interaction contrasts provided by the macro \%PreviewAMMI. This \%IC2by2T macro should only be used after one has determined that interaction is present in the data by the \%FitAMMImodel macro, in order to provide some control over the experimentwise multiple comparison error rate.

The macro \%ContrastAMMI is designed to test user specified interaction contrasts in the AMMI model with only one interaction term. Users are able to specify interaction contrasts of the form $\mathbf{H} \boldsymbol{\alpha}=\mathbf{0}$ and/or $\mathbf{G} \gamma=\mathbf{0}$ as described in Section 2.3. For specified contrast matrices, $\mathbf{H}$ and $\mathbf{G}$, a simulated pvalue and two simulated critical points, $90 \%$ and $95 \%$, are provided with the test statistic. The built-in simulation module generates 4999 random matrices to calculate $\Lambda$ which is defined in Section 2.3. The simulation method can be viewed as a parametric bootstrap technique providing a simulated null distribution of $\Lambda$ corresponding to the values of $b, t, p, q$, and $\delta=\lambda_{1} / \sigma$.

\subsection{Least Squares Means}

Least squares means for Tukey's model when $\hat{\tau}$ is equal to some known value $\tau$ when viewed as regressing on the $\hat{\tau}$ 's are defined as

$$
L S M_{j}=\hat{\mu}+\tau+\hat{\beta}_{j}+\hat{\lambda} \tau \hat{\beta}_{j}=\hat{\mu}+\hat{\beta}_{j}+\left(1+\hat{\lambda} \hat{\beta}_{j}\right) \tau, \quad j=1,2, \cdots, b .
$$

The macro \%LSMTukey is designed to calculate least squares means for Tukey's model at a specified location along the factor 1 axis.

Least squares means for Mandel's model when $\hat{\tau}$ is equal to some known value $\tau$ when viewed as regressing on the $\hat{\tau}$ 's are defined as

$$
L S M_{j}=\hat{\mu}+\tau+\hat{\beta}_{j}+\tau \hat{\gamma}_{j}=\hat{\mu}+\hat{\beta}_{j}+\left(1+\hat{\gamma}_{j}\right) \tau, \quad j=1,2, \cdots, b
$$

and an approximate standard error of each of the least squares means at $\tau$ is given by 


$$
\sqrt{\hat{\sigma}^{2}\left(\frac{1}{t}+\frac{\tau^{2}}{\sum_{i=1}^{t} \hat{\tau}_{i}^{2}}\right)}
$$

Approximate studentized t-statistics to compare two least squares means from Mandel's model when $\hat{\tau}=\tau$ are given by

$$
\operatorname{Tdiff}_{\left(j, j^{*}\right)}=\frac{L S M_{j}-L S M_{j^{*}}}{\sqrt{2 \hat{\sigma}^{2}\left(\frac{1}{t}+\frac{\tau^{2}}{\sum_{i=1}^{t} \hat{\tau}_{i}^{2}}\right)}}, \quad j, j^{*}=1,2, \cdots, b .
$$

The macro \%LSMandel is designed to calculate and compare least squares means for Mandel's bundle-ofstraight-lines model at a specified location along the factor 1 (factor 2) axis.

\section{EXAMPLES}

To illustrate results from the AMMI macros, data on the growth rate of sorghum plants is used: See Milliken and Johnson (1989). The experimenter has 20 growth chambers and conducts an experiment to study the effects of five temperature levels combined with each of four humidity levels on the growth rate of sorghum plants. Ten sorghum plants of the same species are placed in each of the 20 growth chambers and temperature by humidity treatment combinations are randomly assigned to the 20 chambers. Heights were measured after growing for a month. The mean heights in centimeters of ten plants from each growth chamber are given in Table 2. According to the Type I interaction plots and the Type II interaction plots shown in Figure 5 and Figure 6, there is interaction between humidity and temperature. The scree plot shown in Figure 7 suggests that one interaction term in the AMMI model would be sufficient to model these two-way data.

Tukey's single-degree-of-freedom test for nonadditivity is provided in Table 3 ( $F=28.40, p=.0002$ ) and it shows that there is significant interaction in these data. Figure 8 shows the fit of Tukey's model to the data. This is a Type II interaction plot along with the estimated regression lines given by Tukey's model with a line for each temperature. The estimated regression equations are also shown for each temperature level at the bottom of the plot. The concurrency test between Tukey's and Mandel's model is given in Table $4(F=4,77, p=.0343)$ and it suggests that Mandel's model describes the interaction significantly better than Tukey's model. Mandel's test for interaction ( $F=17.99, p=.0005$ ) reveals that there is significant interaction in these data. Figure 9 shows the fit of Mandel's model to the data. Estimation of the estimated regression lines in Figure 9 would seem to indicate that there is very little interaction between temperature levels 50, 60, and 70 and all humidity levels because the three lines associated with these temperature levels are nearly parallel to each other. The estimate of the experimental error variance from Mandel's model is 5.007969 with 8 degrees of freedom. Table 5 shows the results of fitting an AMMI model with one interaction term. The value of the pseudo F-test statistic for interaction is 4.07 and its p-value is 0.1058 . The estimate of the experimental error variance is 10.694815 with degrees of freedom, 3.7062. The largest characteristic root of $\mathbf{Z}^{T} \mathbf{Z}$ and $\mathbf{Z Z}^{T}$ is 360.809876. The estimate $\hat{\lambda}_{1}$ is 18.995 and the estimates of two interaction parameter vectors are $\hat{\boldsymbol{\alpha}}_{1}^{T}=[-.616-.302 .225 .692]$, and

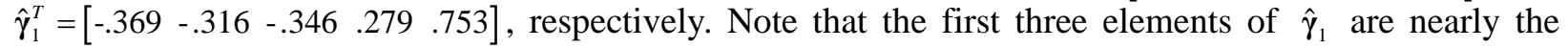


same. Thus, the maximum likelihood estimate of the interaction parameter vector $\gamma_{1}$ also suggests that the lower three temperature levels do not interact with humidity.

Mandel's model suggests there is interaction in these data while the AMMI model suggests there is no interaction in the data. In this example, Mandel's test is likely more powerful than the AMMI test since the form of the interaction test in Mandel's model is more specific. Mandel's model provides a better fit to these data since the estimate of $\sigma^{2}$ from Mandel's model is much smaller than the estimate of $\sigma^{2}$ from the AMMI model. Also note that the sum of squares for interaction from Mandel's model is 360.383249 with 4 degrees of freedom and the pseudo sum of squares for interaction from the AMMI model is 360.809876 with degrees of freedom, 8.2938. An approximate test of whether the AMMI model is significantly better than Mandel's model is given by

$$
F^{*}=\frac{(360.809876-360.383249) /(8.2938-4)}{10.694815}=0.0093
$$

with degrees of freedom 4.2938 and 3.7062. This $F^{*}$ is not statistically significant indicating that the AMMI model is not significantly better than Mandel's model.

Table 6 shows the results of testing all possible pairwise row and pairwise column interaction contrasts based on the estimate of $\sigma^{2}$ given by Mandel's model where one * indicates significance at the $10 \%$ level, two $* *$ indicates significance at the $5 \%$ level, and three *** indicates significance at the $1 \%$ level. There are no *'s in the rows of Table 6 identified by 1,2; 1,3 ; and 2,3 where $1 \equiv 50^{\circ}, 2 \equiv 60^{\circ}$, and $3 \equiv 70^{\circ}$. This also indicates there is no statistically significant interaction between the lower three temperature levels and humidity.

\section{CONCLUSION}

Software for analyzing interaction in two-way experiments is not currently available for wide-spread use. This research involves developing user-friendly statistical software for the analysis of interaction in two-way experiments. A set of SAS ${ }^{\circledR}$ macros, called the AMMI macros, are developed. The AMMI macros provide three stages of analytic tools; pre-analysis for diagnosing interaction, model fitting for selecting a suitable model, and testing interaction contrasts for finding patterns of interaction in the data. The macros also provide many useful graphical displays to help one determine those combinations of the two treatment factors that interact and to help one interpret the results of the analyses. The developed software will soon be available at the author's web-site along with a user's manual. These will allow many researchers to use multiplicative interaction models to describe real-life phenomena. Examples were given to illustrate the use of the macros and how one might interpret the output.

In conclusion, it is hoped that the $\mathrm{SAS}^{\circledR}$ macros developed in this research will provide a data analysis tool to researchers needing to analyze two-way nonreplicated experiments. These methods might accelerate development of new methods related to this area of research.

\section{REFERENCES}

Graybill, F. A. (2000). Theory and Application of the Linear Model. Belmont, California: Wadsworth \& Brooks Cole. (Original work published 1976)

Gollob, H. F. (1968). A statistical model which combines features of factor analytic and analysis of variance techniques. Psychometrika, 33, 73-115.

Hegemann, V., \& Johnson, D. E. (1976). The power of two tests for nonadditivity. Journal of the 
American Statistical Association, 71, 945-948.

Johnson, D. E. (1974). On the moments of the characteristic roots of a noncentral $(2 \times 2)$ Wishart matrix. The Australian Journal of Statistics, 16, 153-155.

Johnson, D. E., \& Graybill, F. A. (1972). An analysis of a two-way model with interaction and no replication. Journal of the American Statistical Association, 67, 862-868.

Mandel, J. (1961). Non-additivity in two-way analysis of variance. Journal of the American Statistical Association, 56, 878-888.

Mandel, J. (1969). The partitioning of interaction in analysis of variance. Journal of Research of the National Bureau of Standards, B. 73B, 309-328.

Marasinghe, M. G., \& Johnson D. E. (1982). A test of incomplete additivity in the multiplicative interaction model. Journal of the American Statistical Association, 77, 869-877.

Milliken, G. A., \& Johnson, D. E. (1989). Analysis of Messy Data, Volume 2: Nonreplicated Experiments (2nd ed.). New York: Chapman \& Hall.

Tukey, J. W. (1949). One degree of freedom for non-additivity. Biometrics, 5, 232-242.

Table 1 An ANOVA table for the AMMI model with $k$ interaction terms

\begin{tabular}{c|c|c}
\hline SV & Degrees of Freedom & Sum of Squares \\
\hline Total & $b t-1$ & $\sum_{i j}\left(y_{i j}-\bar{y}_{. .}\right)^{2}$ \\
T & $t-1$ & $\sum_{i}\left(\bar{y}_{i .}-\bar{y}_{. .}\right)^{2}$ \\
B & $b-1$ & $\sum_{j}\left(\bar{y}_{. j}-\bar{y}_{. .}\right)^{2}$ \\
MI1 & $v_{1}$ & $l_{1}$ \\
MI2 & $v_{2}$ & $l_{2}$ \\
$\vdots$ & $\vdots$ & $\vdots$ \\
MI $k$ & $v_{k}$ & $l_{k}$ \\
Error & $(t-1)(b-1)-\sum_{r=1}^{k} v_{r}$ & $\sum_{i j}\left(y_{i j}-\bar{y}_{i .}-\bar{y}_{. j}-\bar{y}_{. .}\right)^{2}-\sum_{r=1}^{k} l_{r}$ \\
\hline
\end{tabular}

Table 2 Average height of 10 sorghum plants

\begin{tabular}{c|rccc}
\hline \multirow{2}{*}{ Temperature, ${ }^{\circ} \mathrm{F}$} & \multicolumn{4}{|c}{ Humidity, \% } \\
\cline { 2 - 5 } & 20 & 40 & 60 & 80 \\
\hline 50 & 12.3 & 19.6 & 25.7 & 30.4 \\
70 & 13.7 & 16.9 & 27.0 & 31.5 \\
80 & 17.8 & 20.0 & 26.3 & 35.9 \\
90 & 12.1 & 17.4 & 36.9 & 43.4 \\
\hline
\end{tabular}

Table 3 Fitting Tukey's model to the sorghum data

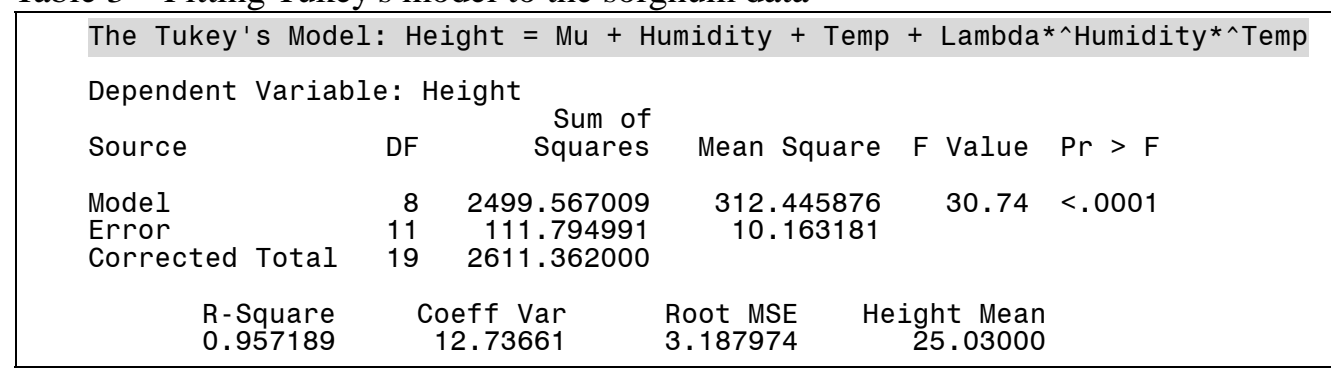


Table 3 (continue)

\begin{tabular}{|c|c|c|c|c|c|c|}
\hline \multicolumn{2}{|l|}{ Source } & \multicolumn{2}{|r|}{$\begin{array}{l}\text { Sum of } \\
\text { Squares }\end{array}$} & Mean Square & e F Value & e $\operatorname{Pr}>\mathrm{F}$ \\
\hline \multicolumn{2}{|c|}{$\begin{array}{l}\text { Humidity } \\
\text { Temp } \\
\text { Tukey's Test for } \\
\text { Nonadditivity }\end{array}$} & $\begin{array}{l}3 \\
4 \\
1\end{array}$ & $\begin{array}{r}2074.298000 \\
136.617000 \\
288.652009\end{array}$ & $\begin{array}{r}691.432667 \\
34.154250 \\
288.652009\end{array}$ & $\begin{array}{r}68.03 \\
3.36 \\
28.40\end{array}$ & $\begin{array}{ll}3 & <.0001 \\
6 & 0.0498 \\
0 & 0.0002\end{array}$ \\
\hline \multicolumn{2}{|l|}{ Parameter } & \multicolumn{3}{|r|}{$\begin{array}{c}\text { Standard } \\
\text { Error }\end{array}$} & \multicolumn{2}{|r|}{$\operatorname{Pr}>|t|$} \\
\hline $\begin{array}{l}\text { Intercept } \\
\text { Humidity } \\
\text { Humidity } \\
\text { Humidity } \\
\text { Humidity } \\
\text { Temp } \\
\text { Temp } \\
\text { Temp } \\
\text { Temp } \\
\text { Temp } \\
\text { Lambda }\end{array}$ & $\begin{array}{l}20 \\
40 \\
60 \\
80 \\
50 \\
60 \\
70 \\
80 \\
90\end{array}$ & $\begin{array}{r}25 . \\
-12 . \\
-6 . \\
5 . \\
13 . \\
-3 . \\
-2 . \\
-0 . \\
2 . \\
3 . \\
0 .\end{array}$ & $\begin{array}{l}3000000 \\
17000000 \\
19000000 \\
5000000 \\
31000000 \\
3000000 \\
5500000 \\
3000000 \\
+2000000 \\
39500000 \\
14272970\end{array}$ & $\begin{array}{l}0.71285276 \\
1.42570551 \\
1.42570551 \\
1.42570551 \\
1.42570551 \\
1.59398722 \\
1.59398722 \\
1.59398722 \\
1.59398722 \\
1.59398722 \\
0.02678193\end{array}$ & $\begin{array}{r}35.11 \\
-8.75 \\
-4.55 \\
3.61 \\
9.69 \\
-1.90 \\
-1.73 \\
-0.02 \\
1.52 \\
2.13 \\
5.33\end{array}$ & $\begin{array}{l}<.0001 \\
<.0001 \\
0.0008 \\
0.0041 \\
<.0001 \\
0.0838 \\
0.1119 \\
0.9853 \\
0.1572 \\
0.0566 \\
0.0002\end{array}$ \\
\hline
\end{tabular}

Table 4 Fitting Mandel's model to the sorghum data

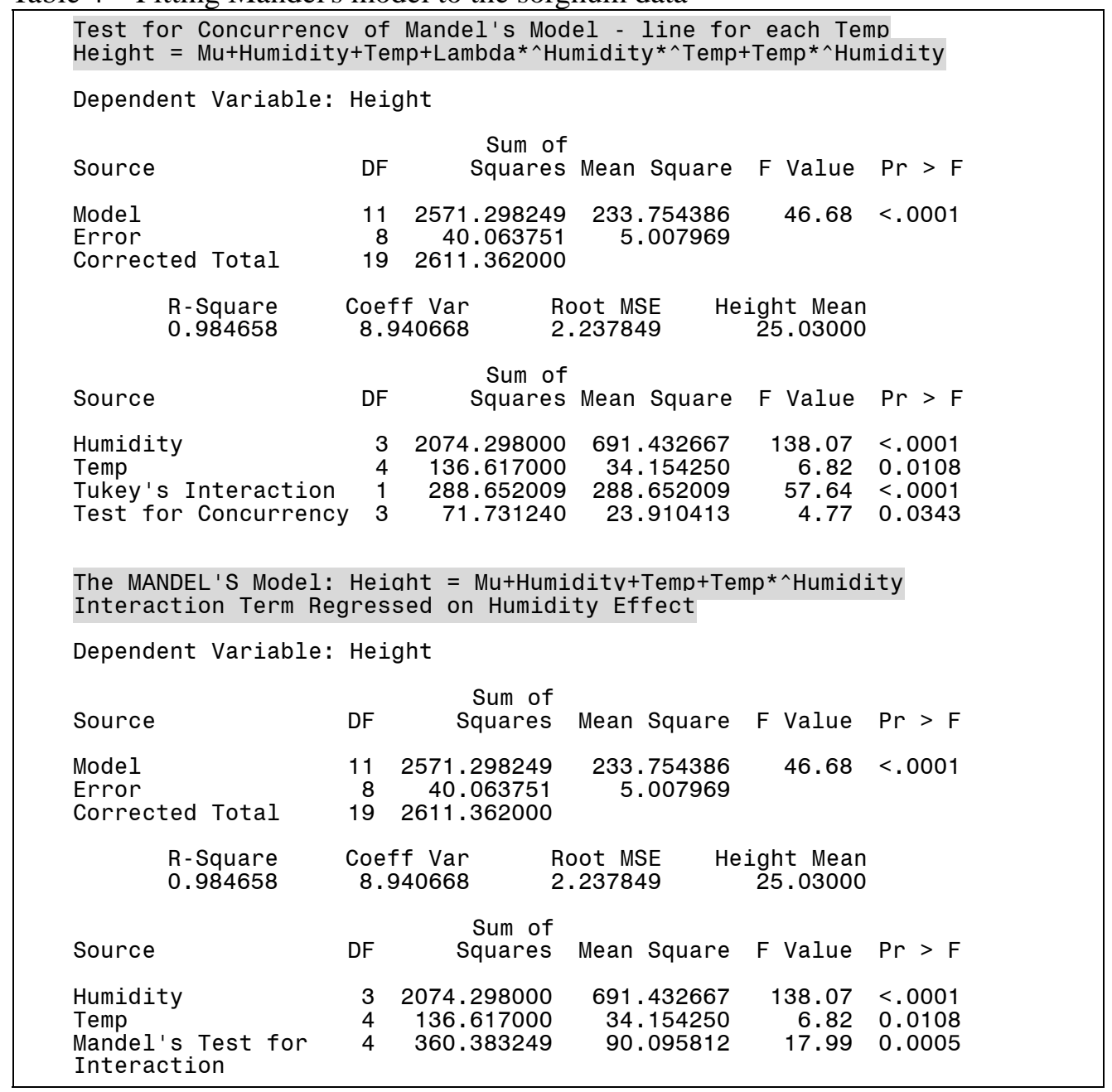


Table 5 Fitting an AMMI model to the sorghum data

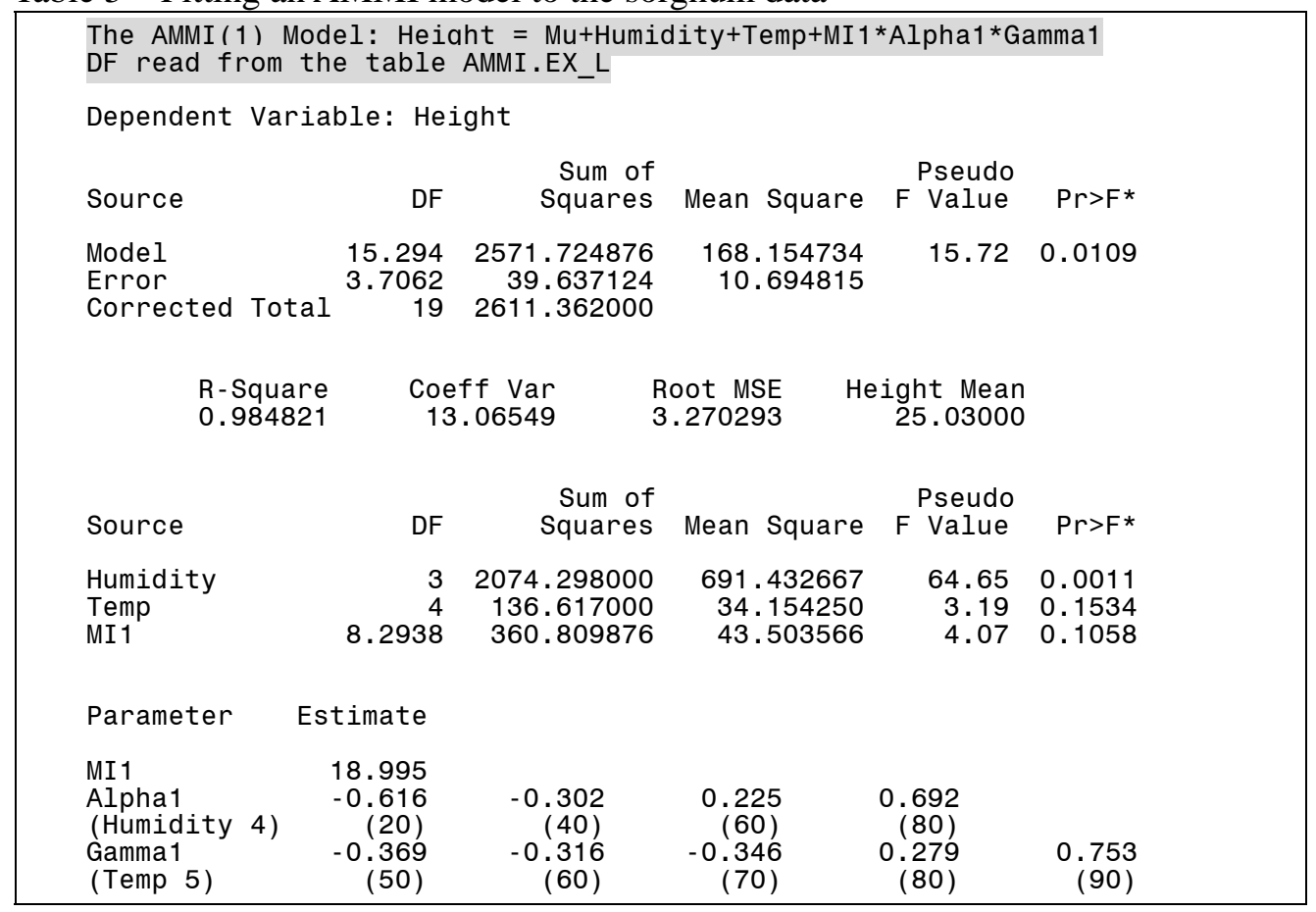

Table 6 Testing all $2 \times 2$ interaction contrasts for the sorghum data

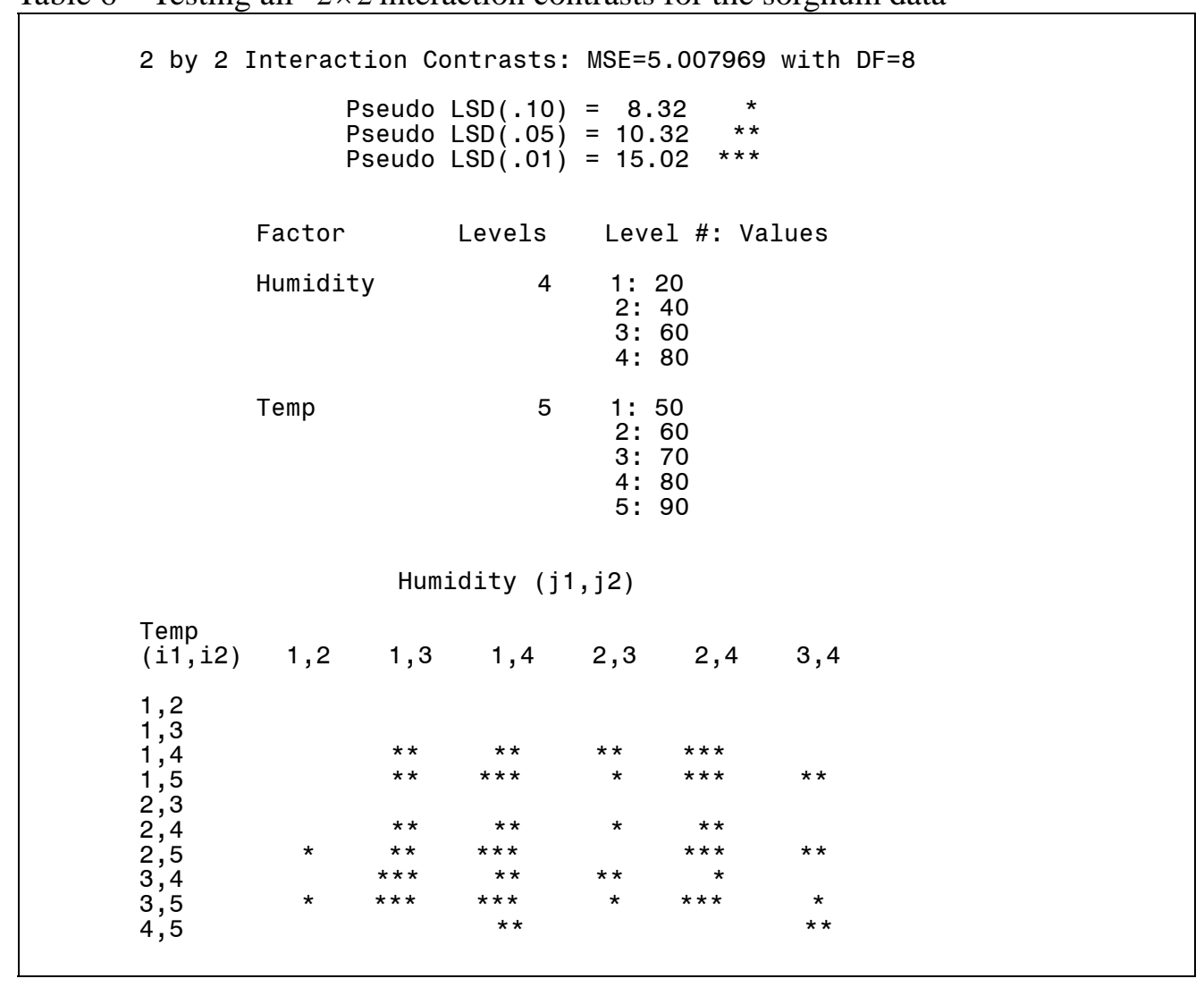




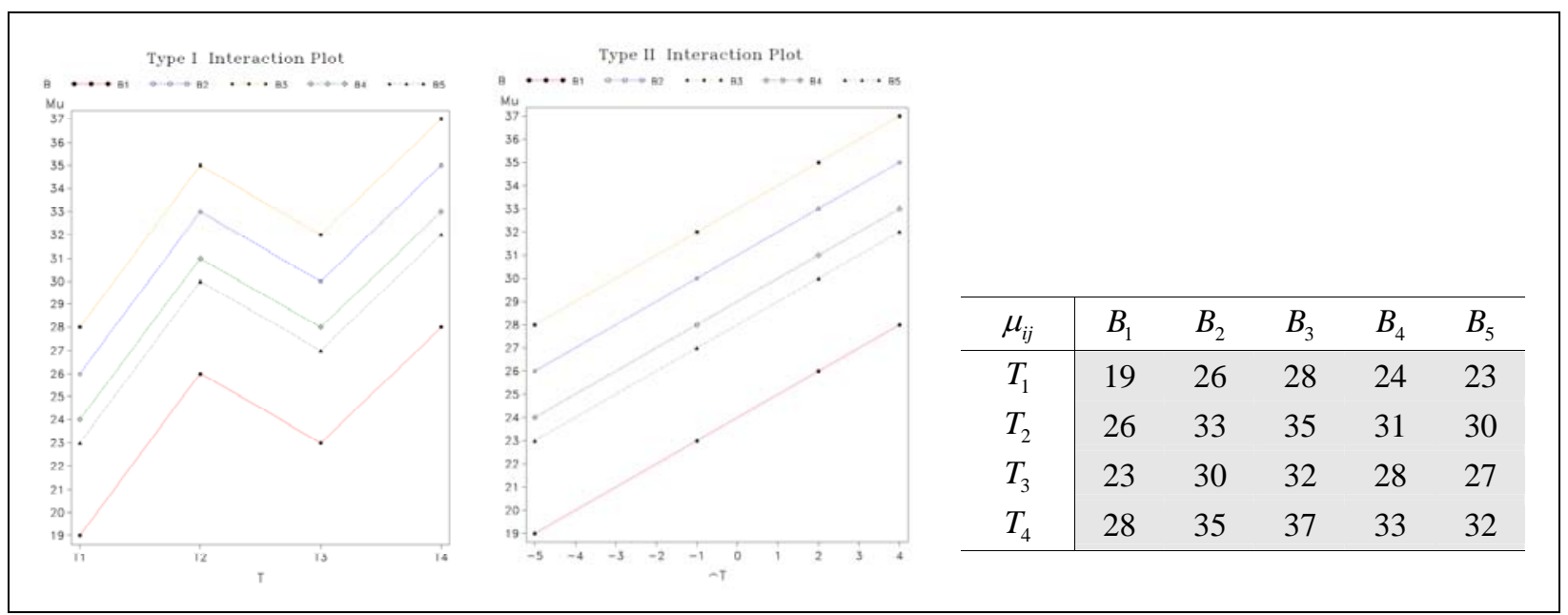

Figure 1 Type I and Type II interaction plots for an additive two-way model

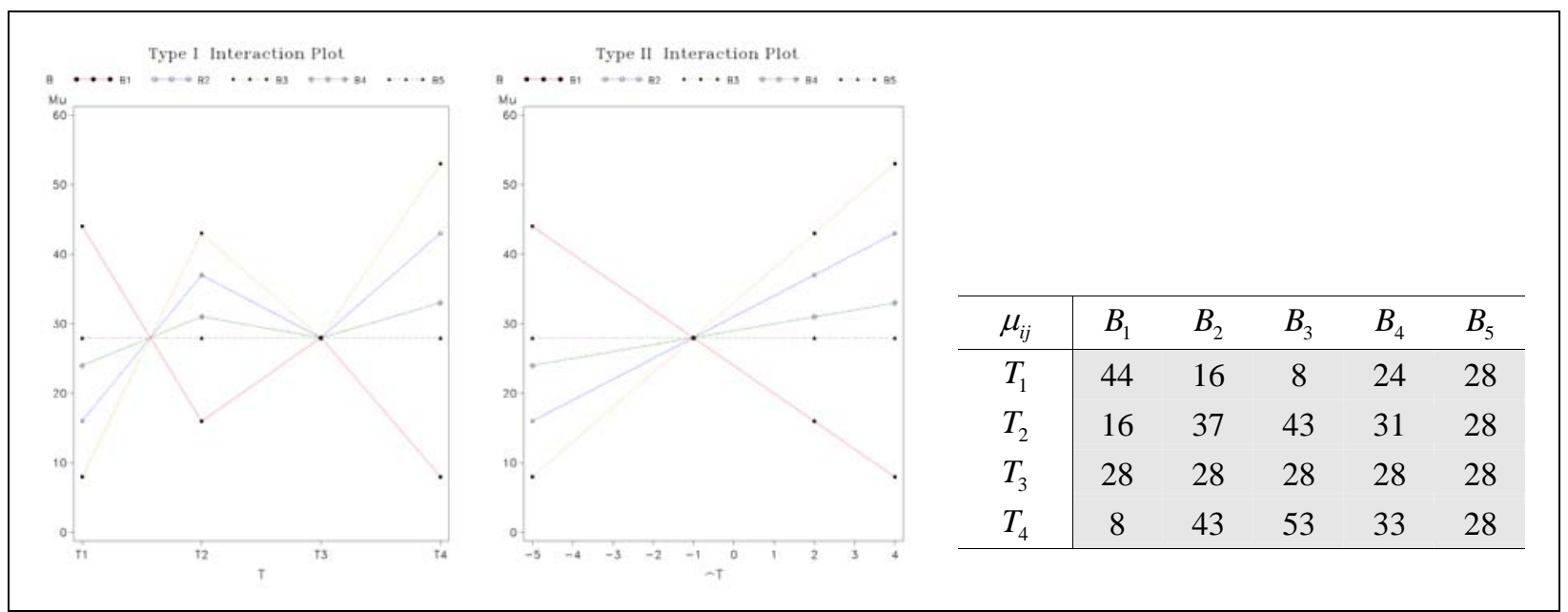

Figure 2 Type I and Type II interaction plots for a Tukey's model

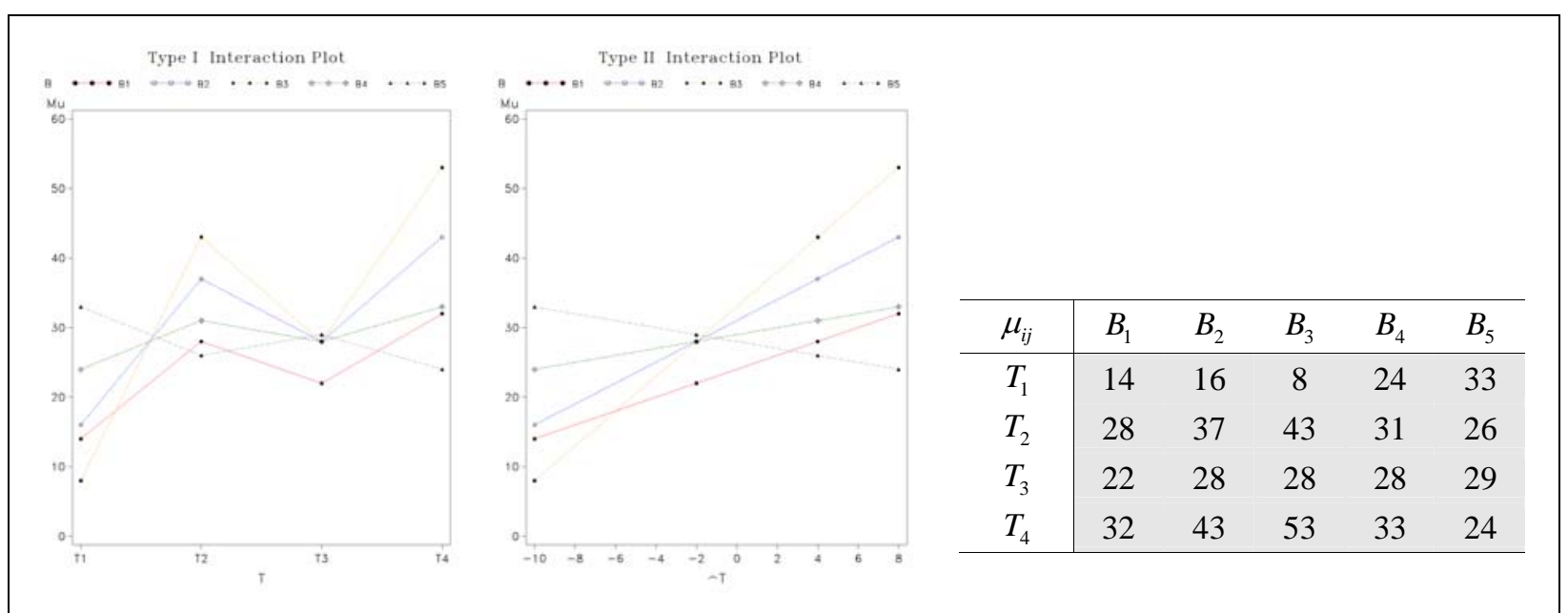

Figure 3 Type I and Type II interaction plots for a Mandel's model 


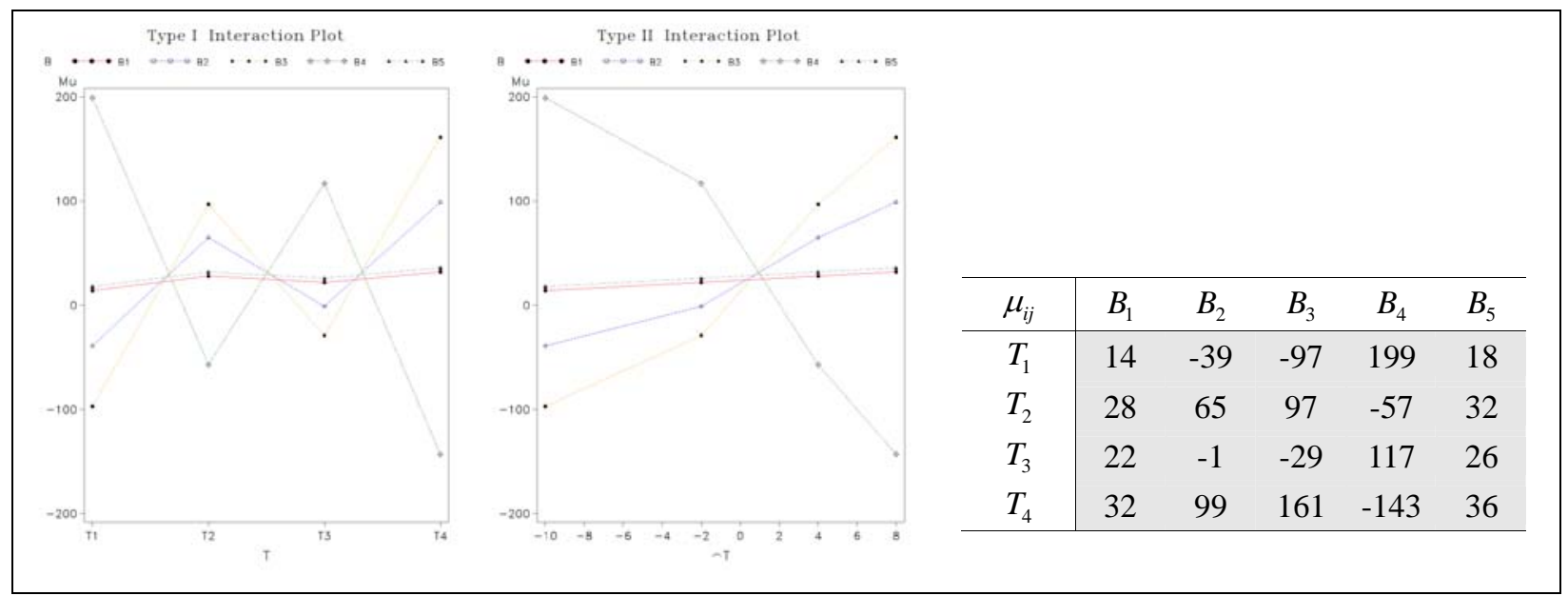

Figure 4 Type I and Type II interaction plots for an AMMI model

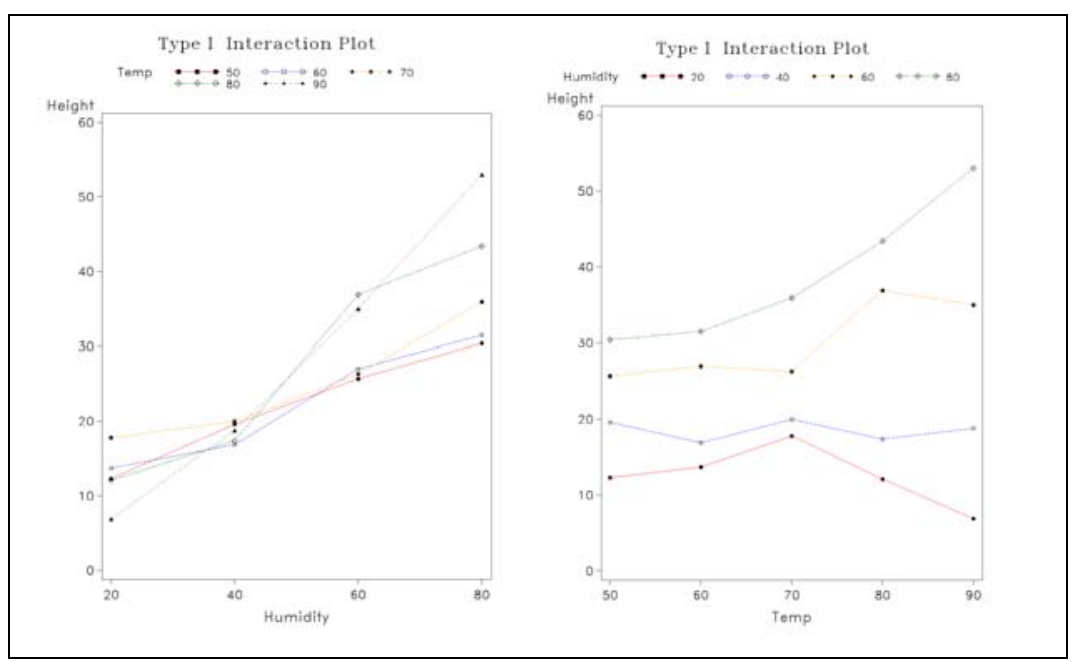

Figure 5 Type I interaction plots for the sorghum data
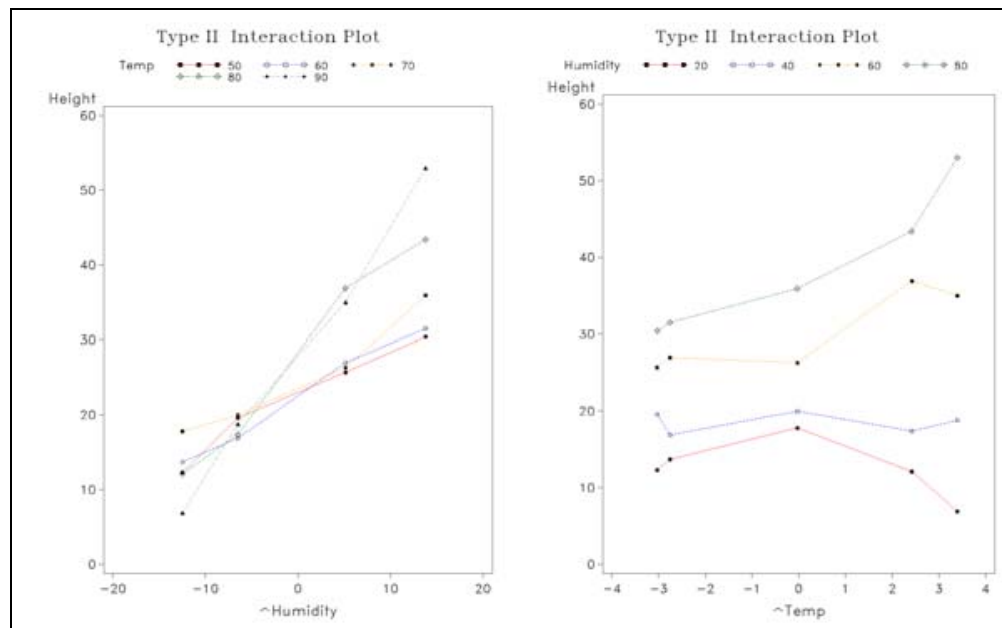

Figure 6 Type II interaction plots for the sorghum data 


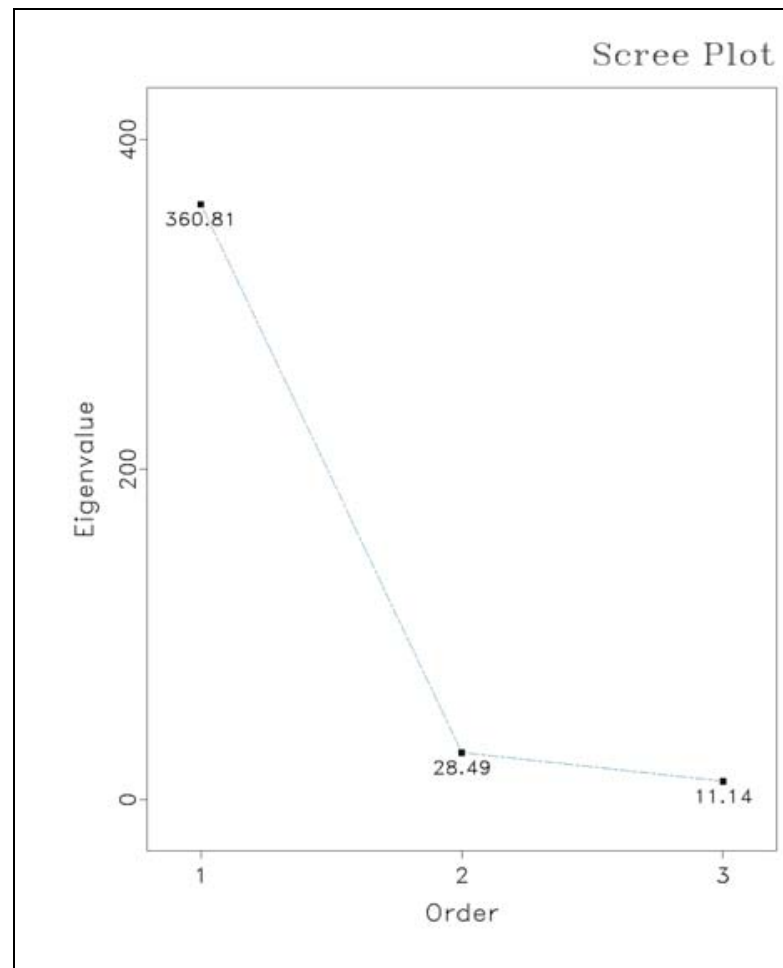

Figure 7 Scree plot for the sorghum data

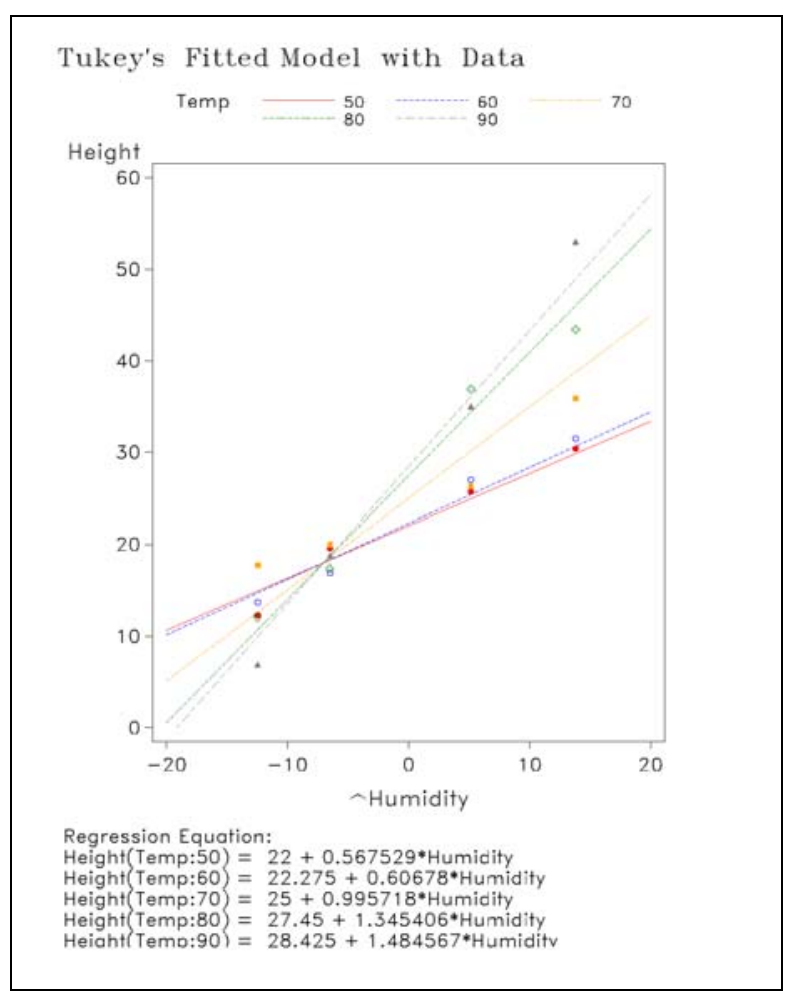

Figure 8 Type II interaction plot and lines predicted by Tukey's model for the sorghum data

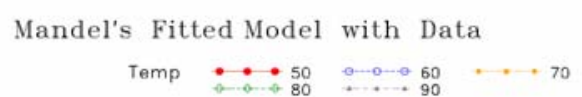

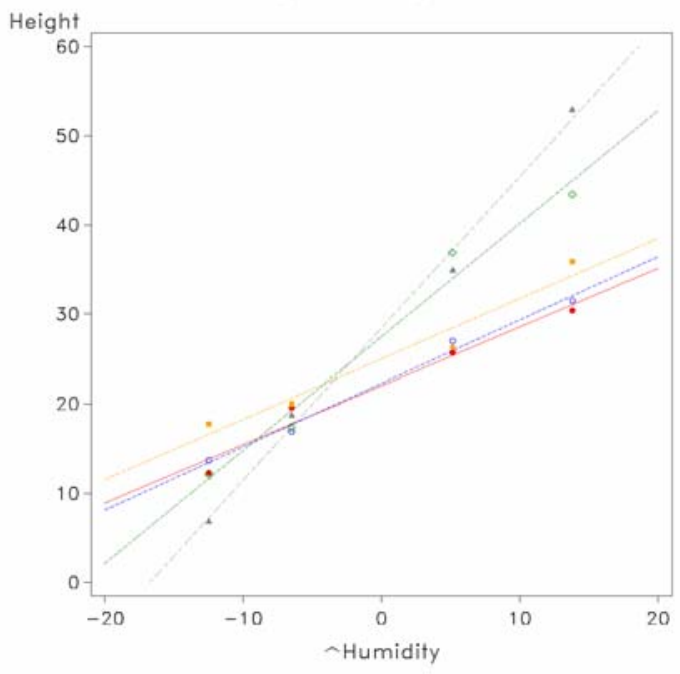

Regression Equation:

Height (Temp:50) $=22+0.654665 *$ Humidity

Height (Temp: 60$)=22.275+0.707577 *$ Humidity

Height Temp: $70=25+0.6736211^{\circ}$ Humidity

Heiaht Temo:90 $=28.425+1.697261 *$ Humiditv

Figure 9 Type II interaction plot and lines predicted by Mandel's bundle-of-straight-lines model for the sorghum data 